\title{
Diagnóstico e conduta cirúrgica nos meningiomas de tubérculo selar
}

Tobias Alécio Mattei' ${ }^{1}$ Ricardo Ramina², Jerônimo Buzetti Milano,3, Paulo Henrique Aguiar ${ }^{4}$, Josias Alécio Mattei ${ }^{5}$

Departamento de Neurocirurgia do Instituto de Neurologia de Curitiba, PR, Brasil Departamento de Neurocirurgia da Faculdade de Medicina da Universidade de São Paulo, SP, Brasil Clínica de Neurocirurgia Pinheiros, São Paulo, SP, Brasil

\section{RESUMO}

Os meningiomas do tubérculo selar, juntamente com os da goteira olfatória, estão entre os tumores mais freqüentes da fossa craniana anterior. Os sintomas característicos são déficits visuais, distúrbios do comportamento, crises epilépticas, anosmia e déficits motores. Devido a seu crescimento lento, o período do início dos sintomas até o diagnóstico tende a ser longo, geralmente excedendo dois anos. Quando o tumor atinge mais de $4 \mathrm{~cm}$ e comprime as estruturas neurovasculares adjacentes, a possibilidade de remoção cirúrgica total é limitada e a chance de melhora na função visual é baixa. O tratamento ideal dos meningiomas de tubérculo selar é a ressecção completa do tumor com descompressão dos vasos e parênquima cerebral adjacentes. Em virtude da escassez de grupos experientes especializados em cirurgia da base do crânio, muitos dos pacientes portadores de meningiomas de tubérculo selar são tratados em alguns centros com procedimentos alternativos, os quais apresentam resultados significativamente piores que as séries cirúrgicas mais recentes publicadas na literatura. Neste artigo, os autores apresentam um panorama geral acerca dos métodos diagnósticos para detecção e avaliação pré-operatória dos meningiomas de tubérculo selar. Também são enfatizados os benefícios do tratamento cirúrgico, o qual, se realizado por uma equipe experiente, pode ser curativo, apresentando menor morbidade que outras modalidades terapêuticas.

\section{PALAVRAS-CHAVE}

Meningiomas. Meningiomas de tubérculo selar.

\section{ABSTRACT}

Diagnosis and surgical management of the tuberculum sellae meningiomas

Meningiomas of tuberculum sellae, together with those from the olfactory groove, are the most frequent anterior skull base meningiomas. The most frequent symptoms in order of frequency are visual loss, mental disorders, epilepsy, anosmia and motor deficits. We stress the still unacceptably long period between the onset of symptoms and the correct diagnosis (in the majority of patients it exceeds two years). When the size of tumor exceeds $4 \mathrm{~cm}$ and the compression on adjacent important neurovascular structures is evident, possibilities of total removal are limited and the chance for visual improvement is low. The ideal treatment of the tuberculum sellae meningiomas is the resection of the tumor, with decompression of the vascular and of the nervous structures. Due to lack of highly specialized and experimented surgical groups, many cases of tubercullum sellae meningiomas are treated by some neurosurgical centers with non-optimal alternative procedures, presenting worse results than recently published surgical series. In this article, the authors provide a general overview of diagnostic methods for detection and correct pre-operative evaluation of tubercullum sellae meningiomas. We also emphasize the benefits of the surgical treatment, which, if managed by experts in skull base neurosurgery, may be curative and present less co-morbidities than other treatment modalities.

\section{KEY WORDS}

Meningiomas. Tubercullum sellae meningiomas.

1 Médico-residente do Departamento de Neurocirurgia do Instituto de Neurologia de Curitiba. Membro do Grupo de Pesquisa em Neuroncologia da Clínica de Neurocirurgia Pinheiros.

2 Neurocirurgião, chefe do Instituto de Neurologia de Curitiba, especialista em cirurgia de Base de Crânio por Klinikum Hannover Nordstadt, Alemanha.

3 Neurocirurgião do Departamento de Neurocirurgia do Instituto de Neurologia de Curitiba. Pós-graduando do Departamento de Neurocirurgia da Faculdade de Medicina da Universidade de São Paulo.

4 Membro do Departamento de Neurocirurgia da Faculdade de Medicina da Universidade de São Paulo. Chefe da Clínica de Neurocirurgia Pinheiros. 5 Membro do Grupo de Pesquisa em Neuroncologia da Clínica de Neurocirurgia Pinheiros. 


\section{Introdução e classificação}

A primeira ressecção completa de um meningioma de tubérculo selar foi realizada em 1916, por Cushing e Eisenhardt, e é mencionada em um artigo publicado em $1929^{1}$. Esses autores classificaram os meningiomas de tubérculo selar como meningiomas supra-selares. Essa classificação é ainda usada por muitos autores 1,4,12,16,2224,35,49. No entanto, uma vez que essa é uma entidade que pode ser clínica e patologicamente bem definida, esses tumores são, na maioria das vezes, estudados isoladamente daqueles originados na porção posterior da região supra-selar, como, por exemplo, os originados na região dorsal da sela. Apesar disso, alguns autores ${ }^{21}$ insistem que os meningiomas de tubérculo selar e os meningiomas do diafragma da sela são anatomicamente indistinguíveis e devem ser denominados, ambos, meningiomas de tubérculo selar'.

Em virtude da dificuldade de definição de um quadro clínico específico, Al-Mefty e $\mathrm{Smith}^{3}$ classificaram como meningiomas do tubérculo selar àqueles originários da porção anterior da região supra-selar, tanto aqueles com origem no tubérculo quanto aqueles com origem na lâmina esfenoidal, na fissura quiasmática e no diafragma da sela túrcica, embora eles recomendem que esse último seja considerado separadamente.

\section{Incidência}

Os meningiomas de tubérculo selar, em conjunto com aqueles da goteira olfatória, são os meningiomas mais freqüentes na fossa anterior. Os meningiomas de tubérculo selar estão entre 5,5\% e 10\% dos meningiomas intracranianos $8,24,27,36,44$. Esses tumores aparecem mais freqüentemente na quinta década de vida e com nítida predominância em mulheres.

\section{Sinais e sintomas}

Os meningiomas de tubérculo selar são caracterizados pela "síndrome quiasmática" (atrofia óptica primária, com perda do campo visual bitemporal, em pacientes com sela túrcica normal), descrita por Holmes e Sargent ${ }^{18}$ e, posteriormente, divulgada por Cushing e Eisenhardt ${ }^{8}$. Em razão de sua origem no tubérculo selar, esses tumores deslocam o quiasma superior e posteriormente e causam sintomas mais precocemente que os tumores da goteira olfatória.
Os sintomas mais freqüentemente observados são, em ordem de freqüência: déficit visual, distúrbios mentais, epilepsia, anosmia e déficits motores ${ }^{43}$. Perda visual de algum grau foi observada em $98 \%$ dos pacientes; atrofia óptica em $78 \%$, hemiparesia em $15 \%$, anosmia em 11\% e síndrome de Foster-Kennedy (caracterizada por atrofia óptica primária e escotoma central no lado da lesão e edema de papila no lado oposto) em $5 \%$ dos pacientes.

Normalmente, esses tumores manifestam-se mediante perda insidiosa, progressiva e assimétrica da visão, iniciando com decréscimo da acuidade visual unilateral ou redução concêntrica do campo visual, seguida por perda bilateral da visão. Eventualmente, a perda visual pode ser intermitente e variar ao longo da história da doença ${ }^{10,14,33,46}$.

No exame neuroftalmológico, são freqüentemente encontrados: distúrbio assimétrico e incongruente do campo visual, atrofia óptica primária com escotoma central, distúrbio bitemporal e assimétrico do campo visual e, excepcionalmente, perda visual bitemporal simétrica. O distúrbio visual pode piorar com a gravidez e melhorar sensivelmente após o parto ${ }^{4,10,46,53}$, reforçando a dependência hormonal dos meningiomas em geral. Edema de papila é menos freqüente e ocorre mais tardiamente 4 .

Cefaléia é o segundo sintoma mais freqüente - encontrada em $50 \%$ dos casos $^{3}$ e predomina na região frontal ou retroorbital ${ }^{10,9,46}$. Distúrbios mentais (perda de memória, alterações de personalidade, depressão e ansiedade) ocorrem em $10 \%$ dos pacientes ${ }^{44,47}$. Eventualmente, o quadro pode iniciar-se com crises convulsi$\operatorname{vas}^{32}$. Déficits motores são menos freqüentes ${ }^{4}$.

Quando crescem para a região posterior, os tumores do tubérculo selar comprimem o hipotálamo e a hipófise. Sinais de insuficiência hipofisária são raros no pré-operatório e ocorrem mais tardiamente ${ }^{44,47}$. Entretanto, hiperprolactinemia decorrente de distúrbio hipotalâmico tem sido relatada ${ }^{4,41,42}$.

Anosmia não é freqüente nos meningiomas de tubérculo selar. Poppen ${ }^{35}$ afirmou que anosmia é um sintoma inicial nos meningiomas de goteira olfatória, enquanto os distúrbios visuais aparecem mais tardiamente, em oposição aos meningiomas de tubérculo selar, os quais se apresentam inicialmente com distúrbios visuais e somente mais tarde com anosmia.

\section{Diagnóstico por imagem}

\section{Radiografia simples}

Os meningiomas do tubérculo selar normalmente provocam densa hiperostose, que pode ser vista na 
radiografia simples de crânio em projeção ântero-posterior e de perfil; pode haver aspecto "em pincel" com sua implantação na base do crânio ${ }^{48}$. Outra alteração característica desses tumores é a compressão em "bolha" do osso cortical superior das células etmoidais posteriores e do seio esfenoidal anterior, o que, normalmente, é associado à hiperostose no plano esfenoidal, no plano do tubérculo selar ou à hiperostose "em pincel".

\section{Angiografia carotidea}

A angiografia carotídea é um exame importante na avaliação pré-operatória dos meningiomas de tubérculo selar. Fornece a localização das artérias carótidas e cerebrais anteriores em relação à superfície tumoral e, também, permite verificar o possível envolvimento destas pela massa tumoral. Esses tumores demonstram as características angiográficas de lesões subfrontais (figura 1$)^{49}$ :

- elevação da artéria cerebral anterior e de seus ramos. Esses vasos estão esticados e empurrados superiormente, formando um arco com concavidade voltada para a base do crânio. O tamanho do arco indica o tamanho do tumor naquele ponto;

- a artéria cerebral anterior é centrada ou deslocada (deslocação rotatória) em relação à linha mediana, dependendo da localização mediana ou paramediana do tumor;

- o sifão carotídeo é deslocado inferiormente (arco fechado);

- a bifurcação da artéria carótida interna é deslocada posteriormente (a linha perpendicular ao nível da bifurcação passa posteriormente à porção intracavernosa da carótida).

Eventualmente, nos tumores de tubérculo selar que crescem posteriormente, a artéria basilar pode estar deslocada.

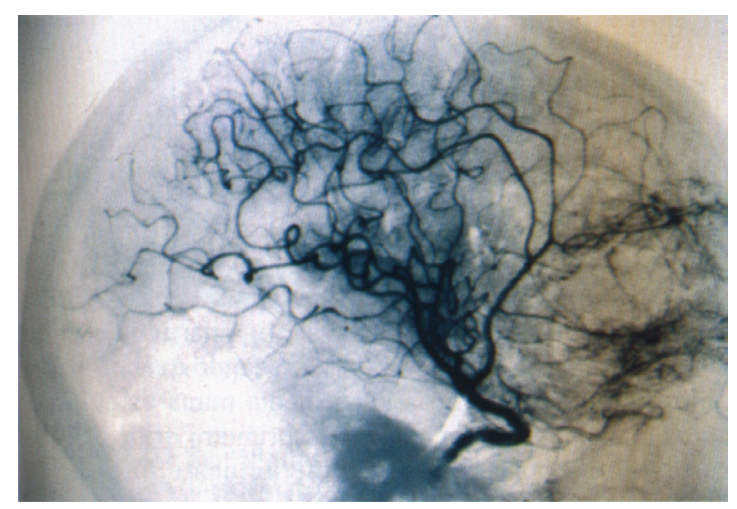

Figura 1-Angiografia cerebral mostrando a vascularização de meningioma de tubérculo selar que desvia a artéria cerebral anterior.
A artéria oftálmica irriga pelo menos parcialmente esses tumores, através de seus ramos etmoidais posteriores, e está hipertrofiada mesmo na ausência de retenção de contraste pela neoplasia. Para definir a artéria oftálmica como anormal, é necessária a visualização de seus ramos etmoidais na junção destes com os ramos meníngeos, no assoalho orbital ${ }^{49}$.

O envolvimento das grandes artérias pela neoplasia pode ser observado nas angiografias por meio de constrições locais na parede dos vasos e, até mesmo, obstruções em alguns casos. Nas fases arteriolar e venosa, os meningiomas de tubérculo selar normalmente apresentam retenção homogênea do contraste, o que determina o tamanho exato da neoplasia. Na fase venosa, a veia septal usualmente está deslocada superiormente, e a porção anterior da veia cerebral interna pode estar deslocada posteriormente e com seu arco aumentado ${ }^{49}$.

\section{Tomografia computadorizada}

A tomografia computadorizada (TC) sem contraste permite o diagnóstico de meningioma em $63 \%$ dos casos e, após a injeção de contraste, esse índice sobe para 90\%, relatados na era pré-ressonância magnética ${ }^{32}$. Tumores com diâmetros menores que $1 \mathrm{~cm}$, principalmente aqueles de localização parasselar, orbital ou parassagital, podem não ser detectados pela $\mathrm{TC}^{27}$. Na fase sem contraste, os meningiomas apresentam-se como lesões hiperdensas em $75 \%$ dos casos, hipodensas em $14,4 \%$ e, ocasionalmente, isodensas. A TC é mais sensível que a radiografia simples para a detecção de depósitos de cálcio e mostra calcificações em $10 \%$ a $26 \%$ dos meningiomas ${ }^{52}$. As calcificações variam de pequenos pontos a grandes áreas formadas pela confluência de psamomas.

Após a injeção de contraste, esses tumores, quando não muito calcificados, normalmente apresentam retenção homogênea do contraste, com contorno nítido e regular, algumas vezes lobulado, e uma grande área de contato com a dura-máter na região do plano esfenoidal. Em 15\% dos pacientes com meningiomas, podem ser achados necrose, sangramentos, lesões císticas e lipomatose ${ }^{39}$. A TC mostra áreas hipodensas ao redor do meningioma, o que significa edema cerebral em $60 \%$ a $70 \%$ dos $\operatorname{casos}^{11,30,43,52}$. Outras lesões, como encefalomalacia, desmielinização, encistamento de liquor, cistos aracnóideos, cistos protéicos e gliomas, podem apresentar-se também como áreas hipodensas ao redor dos meningiomas ${ }^{40}$.

\section{Ressonância nuclear magnética}

A ausência de artefatos causados pelo osso e a possibilidade de melhorar a visualização dos meningiomas 
com contraste paramagnético (figura 2) fazem da ressonância magnética $(\mathrm{RM})$ o exame que fornece a maior quantidade de informações em tumores intracranianos, permitindo a identificação de lesões que passaram despercebidas na $\mathrm{TC}^{6,4,54}$.

Freqüentemente, os meningiomas são isointensos em relação ao cérebro, em T1 e T2. A seqüência de pulso com saturação parcial e recuperação invertida (T1-pesada) permite melhor resolução espacial e mostra a localização extra-axial de alguns tumores, bem como seus efeitos de massa e deslocamentos estruturais com compressão da substância branca ${ }^{16}$. O edema ao redor dos tumores pode ser facilmente detectado na seqüência T2-pesado. A RM também mostra melhor os deslocamentos, oclusões e envolvimento de vasos pelo tumor que a TC e, possivelmente, melhor que a angiografia convenciona $^{38,54}$. Em relação à TC, a RM é inferior na identificação de calcificações e hiperostoses associadas ao meningioma ${ }^{5,38}$.

A injeção de contraste paramagnético (gadolínioDTPA) permite melhor visualização dos meningiomas e a distinção entre meningiomas de tubérculo selar e outras lesões supra-selares. Apesar dos recentes avanços em imagens neurodiagnósticas, pode ser difícil diferenciar, de forma pré-operatória, um meningioma de tubérculo selar de um macroadenoma pituitário. A RM tem suplantado a tomografia e é a primeira escolha para lesões selares e supra-selares. Entretanto, a RM sem contraste não é confiável para distinguir meningiomas de tubérculo selar de macroadenomas pituitários. A diferenciação acurada entre esses dois diagnósticos de massas supra-selares é importante, pois, enquanto um meningioma sempre requer craniotomia, a via transesfenoidal é preferível na remoção de macroadenomas pituitários.

Três características distinguem os meningiomas de tubérculo selar dos macroadenomas pituitários: contrastação homogênea e brilhante com gadolínio, em oposição à contrastação escassa e heterogênea; epicentro tumoral mais supra-selar que selar; extensão paralela à base dural.

Cada um desses achados pode ser sutil, mas o exame cuidadoso de imagens da região parasselar em secções coronal e sagital, de alta qualidade e contrastadas com gadolínio, permite um correto diagnóstico pré-operatório.

\section{Tratamento cirúrgico}

Os tumores frontobasais têm sido operados pelas vias unilateral e bifrontal. Muitos autores ${ }^{3,19,25,27,33,35,45}$ preferem realizar craniotomia lateralizada direita, com menor ou maior extensão por meio de ressecções ósseas (frontal, frontotemporal ou pterional, com ou sem ressecção do arco supra-orbital), e através da via frontal ${ }^{25,27,32,35,45}$ ou através do arco esfenoidal ${ }^{5,19}$. Na via bifrontal ${ }^{24,29}$, o acesso é feito através da fissura inter-hemisférica.

Alguns autores recomendam a craniotomia frontotemporal, com ressecções não-radicais para tumores parasselares e radiocirurgia para tumores residuais e recorrentes $^{37,20}$, enquanto outros afirmam que a remoção radical dos tumores pode resultar em menores taxas de recorrência sem aumentar as complicações cirúrgicas ${ }^{34}$. Operações radicais em tumores perto da haste hipofisária são contra-indicadas ${ }^{15,17}$.

A decisão sobre a abordagem dos tumores frontobasais deve ser baseada principalmente em sua localização em relação aos eixos ântero-posterior e axial. Os meningiomas de tubérculo selar com dimensões maiores são mais bem atingidos por meio de craniotomia bifrontal, enquanto os tumores menores, especialmente aqueles posicionados mais posteriormente, são mais
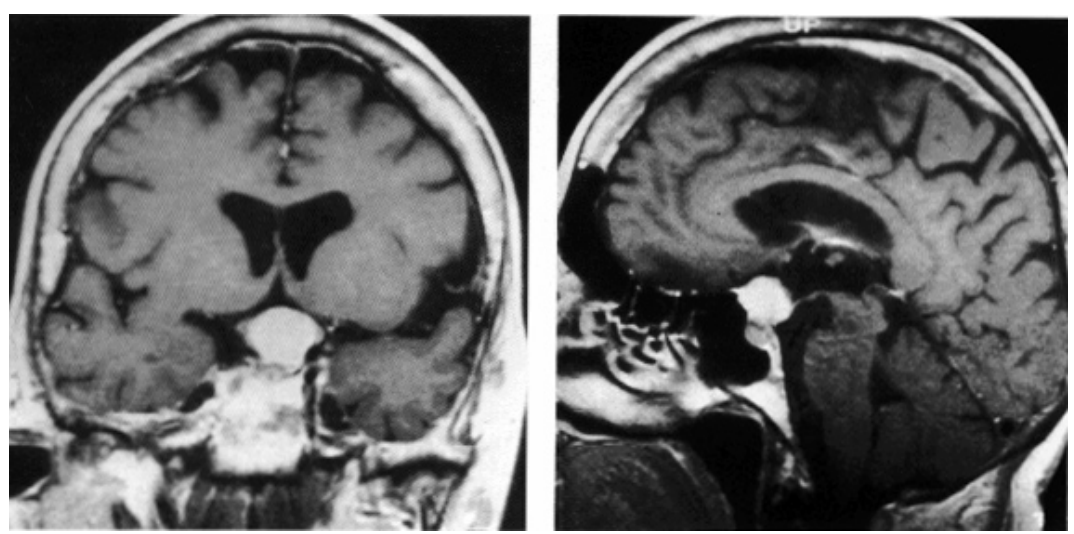

Figura 2 - Cortes coronal e sagital de RM demonstrando meningioma de tubérculo selar. 
bem atingidos por meio de craniotomias unilaterais. Os autores têm atualmente preferido a ressecção por meio de craniotomia unilateral frontolateral (subfrontal com pequena extensão lateral) para a maioria dos tumores, mesmo os maiores (figura 3 ).

A extensão e duração dos sintomas visuais, o envolvimento do complexo da artéria cerebral anterior e o tamanho do tumor são fatores importantes que afetam a cirurgia ${ }^{12}$. O tratamento ideal dos meningiomas frontobasais é a ressecção completa do tumor, com descompressão de vasos e do tecido nervoso e ressecção da dura-máter comprometida. No entanto, antes de tomar a decisão, deve-se considerar os riscos e benefícios, uma vez que, na maioria das vezes, o tumor cresce lentamente. Dessa maneira, é inaceitável que um paciente idoso com exame neurológico normal, por exemplo, após uma cirurgia radical, apresente graves seqüelas, como perda visual importante, para o resto de sua curta vida.

A

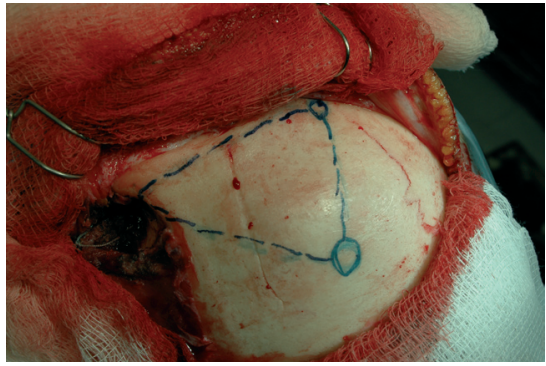

B

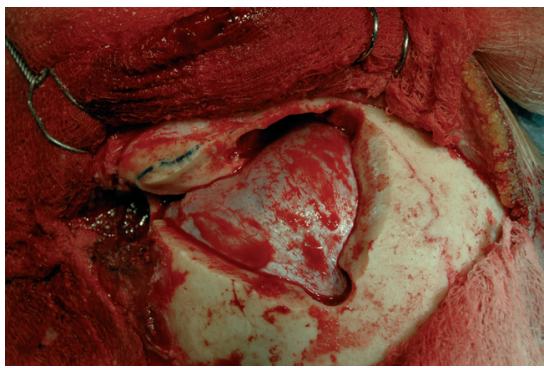

Figura 3 - Fotografias transoperatórias indicando o acesso cirúrgico realizado pelos autores para abordagem de um meningioma de tubérculo selar: A) imagem do acesso subfrontal com pequena extensão lateral advogado pelos autores; B) imagem após realização de craniotomia e antes da abertura dural.

\section{Resultados do tratamento cirúrgico}

Uma vez que os sintomas tendem a ser insidiosos, o período entre o início dos sintomas e o diagnóstico tende a ser longo, e os tumores são diagnosticados já com dimensões maiores. Quando o tamanho do tumor excede $4 \mathrm{~cm}$, e a compressão de importantes estruturas neurovasculares é evidente, as possibilidades de remo- ção completa são limitadas e a chance de melhora da função visual é baixa.

Os distúrbios visuais observados no pré-operatório melhoram em $25 \%$ a $79 \%$ dos pacientes após tratamento cirúrgico $^{2-4,10,14,22-24,32,47,51}$. Diversos fatores interferem favoravelmente no prognóstico pós-operatório da visão, entre eles, duração da sintomatologia inferior a dois anos, tumores menores que $3 \mathrm{~cm}$, perda visual pré-operatória menor que $50 \%$ e presença de papilas normais no exame neurológico $4,24,26,44,47$. Idade avançada e tumores com extensão para o diafragma selar são indicadores de pior prognóstico ${ }^{31}$.

Alguns estudos demonstraram que um tumor de diâmetro maior que $4 \mathrm{~cm}$ é um fator crítico para a função visual. Também se demonstrou que o tamanho da lesão é extremamente importante, pois influencia na quantidade de tumor que pode ser ressecado ${ }^{37}$.

A mortalidade, em séries mais recentes ${ }^{1,3,5,33,38}$, foi reduzida para $5 \%$ a $11 \%$. A ressecção total do tumor visível acontece em $40 \%$ a $100 \%$ dos $\operatorname{casos}^{3,4,8,10,13,14,22-}$ $24,26,32,44,47$. Nos relatos, a razão mais importante para ressecção parcial é a possibilidade de lesão vascu$\operatorname{lar}^{23,24,33}$. Mesmo após ressecção considerada total, ocorre recidiva em $3 \%$ a $25 \%$ dos pacientes, em um período de dois anos e meio ${ }^{7,14,44,31,47}$.

\section{Conclusões}

Nesta revisão, os autores enfatizam o ainda longo período desde o início dos sintomas até o diagnóstico correto dos meningiomas de tubérculo selar. Na maioria dos pacientes, esse período excede dois anos.

Em relação ao diagnóstico, os exames de imagem (principalmente a TC e a RM) são essenciais para a delimitação dos limites tumorais, devendo-se considerar, sempre, a possibilidade de realização da angiografia carotídea para avaliação pré-operatória dos meningiomas de tubérculo selar e suas relações com as artérias carótidas e cerebrais anteriores.

Também, são enfatizados os benefícios do tratamento cirúrgico, o qual, se realizado por uma equipe experiente, pode ser curativo, apresentando menor morbidade que outras modalidades terapêuticas. Entretanto, é importante salientar que, quando o tamanho do tumor excede $4 \mathrm{~cm}$ e há compressão das estruturas neurovasculares adjacentes, a possibilidade de remoção completa é limitada e as chances de melhora da função visual são baixas. 


\section{Referências}

1. AL-MEFTY O: Tuberculum sella and olfatory groove meningiomas. In: SEKHAR LN, JANECKA IP (eds.): Surgery of cranial base tumors. New York: Raven Press, 1993. pp 507-51.

2. AL-MEFTY O, HOLOUBIA, RIFAI A, FOX JL: Microsurgical removal of suprasellar meningiomas. Neurosurgery 16 : 364-72, 1985.

3. AL-MEFTY O, SMITH RR: Tuberculum sellae meningiomas. In: AL-MEFTY O (ed.): Meningiomas. New York: Raven Press, 1991, pp 345-411.

4. ANDREWS BT, WILSON CB: Suprasellar meningiomas: the effect of tumor location on postoperative visual outcome. J Neurosurg 69:523-8, 1988.

5. BENJAMIN V, MCCORNMACK B: Surgical management of tuberculum sellae and sphenoid ridge meningiomas. In: SCHMIDEK HH, SWEET WH (eds.): Operative neurosurgical techniques. Indications methods and results. 3.ed. New York, Grune \& Station, 1995, vol 1, pp 403-13.

6. BRADLEY Jr WG, WALUCH E, YADLEY RA, WYCOFF RR: Comparison of CT and MRI in 400 patients suspected disease of the brain and cervical spinal cord. Radiology 152:695-702, 1984

7. CHAN RC, THOMPSON GB: Morbidity, mortality, and quality of life following surgery of intracranial meningiomas: a retrospective study in 257 cases. J Neurosurg 60:52-60, 1984.

8. CUSHING H, EISENHARDT I: Meningiomas. Their classification regional behaviour life history and surgical end results. 1.ed. Springfield: Charles C. Thomas, 1938, vol 1, pp 224-49.

9. FINN JE, MOUNT LA: Meningiomas of tuberculum sellae and planum sphenoidale: a review of 83 cases. Arch Ophtalmol 92:24-7, 1974

10. DOLENC VV: Direct microsurgical repair of intracavernous vascular lesions. J Neurosurg 58:824-31, 1983.

11. GILBERT JJ, PAULSETH, COTES RK, MALOTT D: Cerebral edema associated with meningiomas. Neurosurgery 12 : 599-605, 1983.

12. GOEL A, MUZUMDAR D, DESAI KI: Tuberculum sellae meningioma: a report on management on the basis of a surgical experience with 70 patients. Neurosurgery 51:135863; discussion 1363-4, 2002.

13. GRANT FC: Meningioma of the tuberculum sellae. Arch Neurol Psychiatry 68:411-2, 1952.

14. GREGORIUS FK, HEPLER RS, STERN WE: Loss and recovery of vision with suprasellar eningiomas. J Neurosurg 42:69-75, 1975

15. GRISOLI F, DIAZ-VASQUEZ P, RISS M, VINCENTELLI F, LECLERCQ TA, HASSOUN $\mathrm{J}$ et al.: Microsurgical management of tuberculum sellae meningiomas. Results in 28 consecutive cases. Surg Neurol 26:37-44, 1986.

16. GUTRIE BL, EBERSOLD MJ, SCHEITHAUER BW: Neoplasms of the intracranial meninges. In: YOUMANS JR (ed.): Neurological surgery. ED 1. Philadelphia, WB Saunders, 1990, vol 5, pp 3250-15.

17. HANSEL G, SEEGER W: The microsurgical treatment of the so-called tuberculum-sellae-meningiomas (author's transl). Acta Neurochir (Wien) 37(1-2):111-23, 1977.

18. HOLMES G, SARGENT P: Suprasellar endotheliomata. Brain 5:518-37, 1927.

19. HUNT WE, SAYERS MP, YASHON D: Tumors of the sellar area. In: YOUMANS JR (ed.): Neurological surgery. Philadelphia, WB Saunders, 1973, vol 3, pp 1414-31.
20. IWAIY, YAMANAKAK, MORIKAWAT, ISHIGURO T, HONDA Y, MATSUZAKA Y et al.: The surgical treatment strategy and results of parasellar meningiomas in the era of radiosurgery. No Shinkei Geka 31:649-55, 2003.

21. JALLO GI, BENJAMIN V: Tuberculum sellae meningiomas: microsurgical anatomy and surgical technique. Neurosurgery 51:1432-39; discussion 1439-40, 2003.

22. JANE JA, MCKISSOK W: Importance of failing vision in early diagnosis of suprasellar meningiomas. Br Med J 2:5-7, 1962.

23. JEFFERSON A, AZZAM N: The suprasellar meningiomas: a review of 19 years's experience. Acta Neurochir (Suppl.) (Vienna) 28:381-4, 1979.

24. KADIS GN, MOUNT LA, GANTI SR: The importance of early diagnosis and treatment of the meningiomas of the planum sphenoidale and tuberculum sellae: a retrospective study of 105 cases. Surg Neurol 12:367-71, 1979.

25. KEMPE LG: Tecnicas neuroquirurgicas. Barcelona, Editorial Cientifico-Medica, 1972, pp 94-103.

26. KONOVOLOV AN, FEODOR SN, FALLER TO, SOKOLOV AF, TCHEREPANOV AN: Experience in the treatment of the parasellar meningiomas. Acta Neurochir (Suppl.) (Vienna) 28:371-2, 1979.

27. MACCARTY CS, PIEPGRAS DG, EBERSOLD MJ: Meningeal tumors of the brain. In: YOUMANS JR (ed.): Neurological surgery. 3.ed. Philadelphia, WB Saunders, 1982, vol 5, pp 2936-66.

28. MARCHELA, BIDZINSKI J, BARCZEWSKAM, DZIEWIECKI C: Meningiomas of sella turcica: some clinical aspects. Neurol Neurochir Pol 30:93-100, 1996.

29. MORLEY TP: Tumors of cranial meninges. In: YOUMANS JR (ed.): Neurological surgery. 3.ed. Philadelphia, WB Saunders, 1973, vol 3, pp 1388-1411.

30. NEW PFJ, ARAONOW S, HESSELINK JR: National Cancer Institute study: evaluation of computed tomography on the diagnosis of intracranial neoplasms. Radiology 136:665-75, 1980.

31. OHTAK, YASUO K, MORIKAWAM, NAGASHIMAT, TAMAKI $\mathrm{N}$ : Treatment of tuberculum sellae meningiomas: a long-term follow-up study. J Clin Neurosci 8: Suppl 1:26-31, 2001.

32. OJEMANN RG: Meningiomas of the basal parapituitary region: technical considerations. Clin Neurosurg 27:233-62, 1980.

33. OJEMANN RG: Surgical management of meningiomas of the tuberculum sellae, olfatory groove, medial sphenoid wing, and floor of the anterior fossa. In: SCHMIDEK HH, SWEET WH (eds.): Operative neurosurgical techniques. Indications Methods and Results. New York, Grune \& Stratton, 1982, vol 1, pp 535-59.

34. OJEMANN RG: Surgical management of meningiomas of olfactory groove meningiomas. In: SCHMIDEK HH, SWEET WH (eds.): Operative neurosurgical techniques. Indications methods and results. 3.ed. New York, Grune \& Stratton, 1995, vol 1, pp 393-401.

35. POPPEN JL: Operative techniques for removal of olfactory and suprasellar meningiomas. Clin Neurosurg 11:1-7, 1963.

36. QUEST DO: Meningiomas: an up-date. Neurosurgery 3: 219-25, 1978.

37. RACO A, BRISTOT R, DOMENICUCCI M, CANTORE G: Meningiomas of the tuberculum sellae. Our experience in 69 cases surgically treated between 1973 and 1993. J Neurosurg Sci 43:253-60, 1999.

38. RANSOHOFF J, NOCKELS RP: Olfactory groove and planum meningiomas. In: APUZZO MLJ (ed.): Brain surgery. Complications avoidance and management. 1.ed. New York, Churchill Livingstone, 1993, vol 1, pp 203-19.

39. RUSSELL EJ, GEORGE AE, KRICHEFF II, BUDZILOVICH $\mathrm{G}$ : Atypical computed tomography features of intracranial 
meningiomas: radiological-pathological correlation in a series of 131 consecutive cases. Radiology 135:673-82, 1980.

40. SCHLEZINGER NS, ALPERS BJ, WEISS BP: Suprasellar meningiomas associated with scotomatous field defects. Arch Ophthalmol 64:908-10, 1967.

41. SHAH RP, LEAVENS ME, SAMAAN NA: Galactorrhea, amenorrha, and hiperprolactinemia as manifestations of prarasellar meningioma. Arch Intern Med 140:1608-12, 1980.

42. SIGEL RM, MESSINAAV: Computed tomography: the anatomic basis of the zone of diminished density surrounding meningiomas. AJR 127:139-41, 1976.

43. SOLERO CL, GIOMBINI S, MORELLO G: Suprasellar and olfactory meningiomas: report on a series of 153 personal cases. Acta Neurochir (Wien) 67:181-94, 1983.

44. SYMON L: Olfactory groove and suprasellar meningiomas. In: KRAYEHBUHL H (ed.): Advances and technical standarts in neurosurgery. New York, Springer-Verlag, 1997, pp 67-91.

45. SYMON L, JAKUBOWSKI J: III meningiomas. Clinical features, technical problems, and results of treatment of anterior parasellar meningiomas. Acta Neurochir (Suppl.) (Wien) 28:367-70, 1979.

46. SYMON L, ROSENSTEIN J: Surgical management of suprasellar meningioma. Part 1: the influence of tumor size, duration of symptoms, and microsurgery on surgical outcome in 101 consecutive cases. J Neurosurg 61:633-41, 1984.

47. TAVERAS JM, WOOD EH: Diagnostic neuroradiology. Baltimore, Willians \& Wilkins, 1976, pp 159-89.

48. TAVERAS JM, WOOD EH: Diagnostic neuroradiology. Baltimore, Willians \& Wilkins, 1976, pp 751-78.
49. TAYLOR SL, BARAKOS JA, HARSH GR 4TH, WILSON CB: Magnetic resonance imaging of tuberculum sellae meningiomas: preventing preoperative misdiagnosis as pituitary macroadenoma. Neurosurgery 31:621-7, 1992.

50. UDVARHELYI GB, WALSH FB: Complications involving the optic nerves and chiasm during the early period after neurosurgical operations. J Neurosurg 19:51-64, 1962.

51. VASSILOUTHIS J, AMBROSE J: Computerize tomography scanning appearances of intracranial meningiomas. An attempt to predict the histological features. J Neurosurg 50:320-7, 1979

52. WEYAND RD, MACCARTY CS, WILSON RB: The effect of pregnancy on intracranial meningiomas occuring about the optic chiasm. Surg Clin N Am 13:1225-33, 2001.

53. ZIMMERMANN RD, FLEMING CA, SAINT-LOUSIS LA, LEE BCP, MANNING JJ, DECK MDF: Magnetic ressonance imaging of meningiomas. Am J Neuroradiol 6:149-57, 1985.

Original recebido em dezembro de 2005

Aceito para publicação em setembro de 2006

\section{Endereço para correspondência}

Tobias Alécio Mattei

Rua Buenos Aires, 630, ap. 502

80250-070 - Curitiba, PR, Brasil

E-mail: tobiasmattei@yahoo.comou

calamus_scriptorius@hotmail.com 